\title{
Russell-Silver Syndrome Presenting as Early Asymmetric IUGR
}

\author{
Haroona Khalil ${ }^{1}$, Hazel Edwards ${ }^{2}$ \& Jane Preston ${ }^{1}$ \\ ${ }^{1}$ James Paget University Hospitals NHS Foundation Trust, Lowestoft Road, Gorleston-on-Sea, Norfolk NR31 6LA, UK, and ${ }^{2}$ Department \\ of Radiography, School of Health and Emergency Professions, University of Hertfordshire, College Lane, Hatfield AL10 9AB, UK
}

\begin{abstract}
We report a case of severe intrauterine growth restriction (IUGR) which was diagnosed as Russell-Silver syndrome (RSS) postnatally. RSS (also known as Silver-Russell syndrome) is one of more than 300 recognised forms of genetic disorder that leads to short stature. Uniparental disomy for chromosome 7 (UPD7), i.e. inheriting two copies of chromosome 7 from the mother, and a change in methylation pattern (biochemical silencing of gene expression) of chromosome 11 are the most frequently associated chromosomal defects. A wide spectrum of appearances and symptoms are associated with the condition. Most characteristics are not easily identifiable with prenatal diagnostic ultrasound. The symptom most likely to be detected sonographically is IUGR. This case seeks to raise awareness of RSS, and encourages clinicians to consider uncommon genetic disorders such as RSS as a possible cause of early asymmetric IUGR.
\end{abstract}

Keywords: Ultrasound, Abdominal Circumference, VSD, Dysmorphia, Short Stature

\section{Case Report}

We report a case of severe intrauterine growth restriction (IUGR) which was diagnosed as Russell-Silver syndrome (RSS) postnatally. A 36 year old Caucasian woman (Gravida 3, Para $2+0$ ) was planned for consultant-led care because of precipitated labour at 36 completed weeks in her previous two pregnancies. The previous pregnancies were not associated with fetal or maternal complications. The woman had no significant medical or surgical problems other than well controlled lower limb psoriasis with associated infrequent use of topical steroid cream. The patient denied smoking and any alcohol or drug intake. There was no family history of chromosomal, congenital or growth anomaly. She was generally healthy, $1.62 \mathrm{~m}$ tall and had a BMI of 22 .

The first obstetric ultrasound scan was performed at 17 weeks gestation and revealed no abnormality. The triple test result was categorised as high risk (1:230). Following counselling, she was offered amniocentesis; this was declined as the woman had already decided to continue with the pregnancy in any circumstance.

At a further scan performed at 19 weeks 5 days gestation, a discrepancy in fetal size was observed, in particular the abdominal circumference fell well below the third centile for normal growth (Fig. 1). Since the fetal heart could not be examined adequately at this attendance, an additional scan was booked for 2 weeks later. At this attendance, the ultrasound scan raised a suspicion of ventricular septal defect (VSD). The patient was referred to a tertiary centre for further sonographic evaluation; however, the suspicion was not confirmed. A few weeks later, another scan in our hospital indicated the possibility of the 'sandal gap' soft marker, but a second opinion by a senior sonographer concluded the fetal feet looked normal. The only persistent abnormality was the significantly reduced fetal size (abdominal circumference below the third centile for normal growth). As the pregnancy

Correspondence: Haroona Khalil, MRCOG, MPHC (Australia), FCPS, James Paget University Hospitals NHS Foundation Trust, Lowestoft Road, Gorleston-on-Sea, Norfolk NR316LA, UK. haroonakhali|@hotmail. com

Ultrasound 2008;16(2):87-90

(c) British Medical Ultrasound Society 2008 progressed, the femur length growth rate also declined (Fig. 2).

From 26 weeks onwards, the pregnancy was managed as 'high risk' and the woman informed of the increased possibility of Down's syndrome due to the high risk triple test result, advanced maternal age and sonographic appearances. Regular ultrasound scans at fortnightly intervals and followup antenatal visits were arranged. Fetal size remained below the third centile for a UK population; estimated fetal weight at 36 weeks was 1179g (Fig. 2). Amniotic fluid volume was considered within the normal range until 35 weeks gestation; ultrasound examinations at 35 and 36 weeks gestation indicated that the amniotic fluid index was below the tenth centile. Doppler ultrasound of the umbilical artery was performed every 2 weeks from 28 weeks gestation; end diastolic flow was always present (Fig. 3 ) and fetal movements were good. In the absence of a karyotype, the pregnancy remained stressful for the woman and her healthcare team.

In view of previous precipitated labour at 36 weeks gestation and reduced amniotic fluid volume in the current pregnancy, labour was induced at 36 weeks gestation. The patient was continuously monitored during labour and delivered a healthy female baby (APGAR scores 7 and 10 at the first and fifth minutes respectively) weighing $1332 \mathrm{~g}$ (below the fourth centile; average birth weight of this centre: $3200 \mathrm{~g}$ ). The paediatric registrar was present at delivery but the baby did not require active resuscitation. The infant developed jaundice on day 1 with a maximum serum bilirubin level 161; full blood count, TORCH screen, chromosomal analysis (46 $X X)$ and cranial ultrasound were normal. The baby stayed in the special care baby unit for 26 days to establish feeding and for close monitoring of her growth.

At 3 months of age, continued monitoring of the infant's growth revealed asymmetry. The upper and lower limbs on the left side were markedly shorter and thinner than on the right. The left hallux was shorter than other toes, which may explain the prenatal suspicion of the 'sandal gap' appearance on ultrasound. The baby's height, weight and head circumference $(40.3 \mathrm{~cm})$ remained under the fourth centile.

The infant was dysmorphic with a triangular face, arched eyebrows, and a naevus flaemmus on the forehead and eyelids. She had an upturned nose with a thin upper lip and 


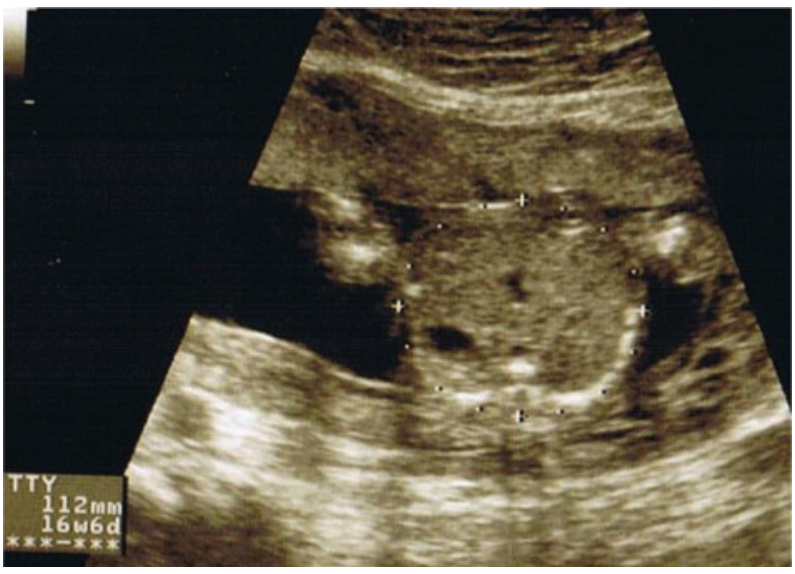

Figure 1. Abdominal circumference taken at 19 weeks and 5 days of gestation.

carp-shaped mouth. Her hands were unremarkable but her feet showed $2 / 3$ skin syndactyly and fourth and fifth toe clinodactyly with a short hallux bilaterally but more marked on the left.

Other growth milestones were unremarkable: the baby smiled at 8 weeks and sat up at approximately 6 months. She passed the routine hearing test and her retinopathy screen was normal. The mother reported that feeding had been difficult and the baby was being supervised by a dietician, paediatric physiotherapist and health visitor.

In the absence of a definitive diagnosis, the baby was referred to a tertiary care centre and was suspected of having RSS. This was confirmed later by genetic analysis and revealed that the infant had the chromosome 11 methylation abnormality. The mother was informed of the good prognosis and advised that management would be individualised depending on any specific needs identified for the child.

In view of the poor postnatal growth observed at 6 months of age, and the prenatal suspicion of VSD, the child was referred for an echocardiogram. A moderately sized perimembranous ventricular septal defect was diagnosed; no abnormal clinical cardiac features that would require intervention were noted. Regular surveillance was recommended and subsequent evaluation indicates that the defect is closing. At the latest review of overall progress, the child was considered 'satisfactory'.

\section{Discussion}

Russell-Silver syndrome (also known as Silver-Russell syndrome) is one of the recognised but very rare forms of IUGR with a genetic origin. It was first described in the 1950s by Silver et al. ${ }^{1}$ and Russell ${ }^{2}$ and is one of more than 300 recognised forms of genetic disorder that lead to short stature.

Prevalence of RSS is variable: it may occur as frequently as $1: 3000$ or as rarely as $1: 100,000$ live births. ${ }^{3}$ RSS represents a phenotype rather than a single specific disorder. ${ }^{4}$ The syndrome is not consistently associated with any particular chromosomal pattern and in some cases the karyotype is normal. The cause of RSS remains unknown with almost all incidences being sporadic, appearing for the first time in individuals with no family history of the condition. ${ }^{5} \mathrm{~A}$ possible familial relationship has been reported in one case where the mother was observed to be only 59 inches. tall, had triangular facies and incurved fifth fingers. ${ }^{6}$ Tanner et al. reported a longitudinal study of 39 cases: none of 61 siblings was affected. $^{7}$

Case reports have however indicated all three possible modes of inheritance: autosomal recessive, autosomal dominant and X-linked. ${ }^{8}$ Duncan et al. ${ }^{8}$ observed the families of 17 cases of RSS and found complete or partial expression of the syndrome in many maternal relatives: one-fifth of the probands analysed had one or more affected relatives. These investigators also demonstrated possible autosomal recessive inheritance in four families and, in two families with affected
BPD

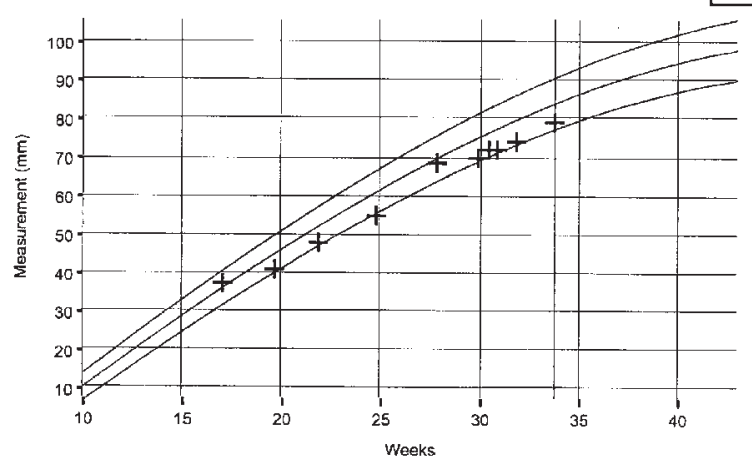

$\mathrm{HC}$

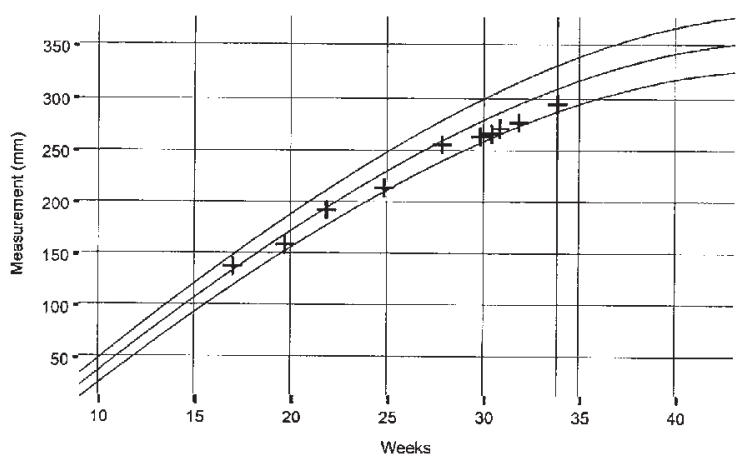

FL

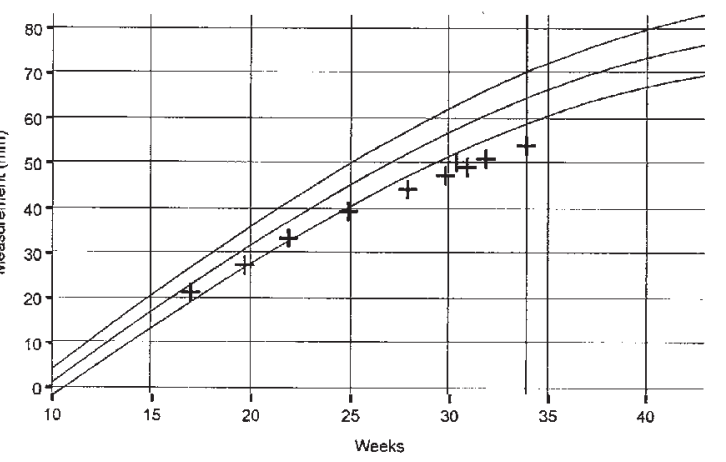

$\mathrm{AC}$

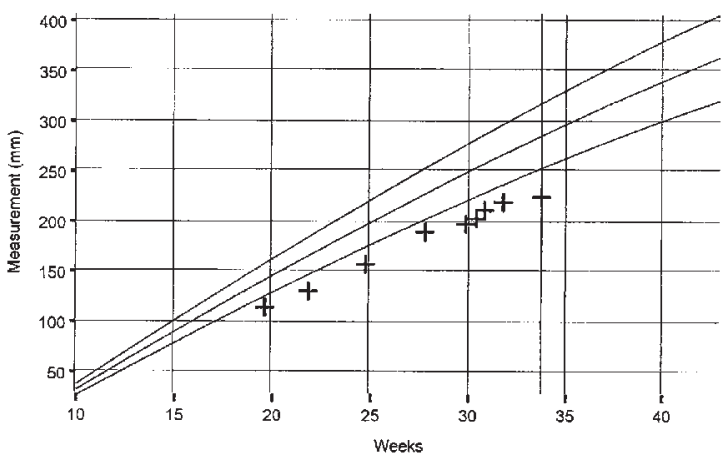

Figure 2. Growth charts (Altman and Chitty, 1994; the third, fiftieth and ninety-seventh centiles) demonstrating acceptable growth of the fetal head but persistently reduced abdominal circumferences and femur lengths. 


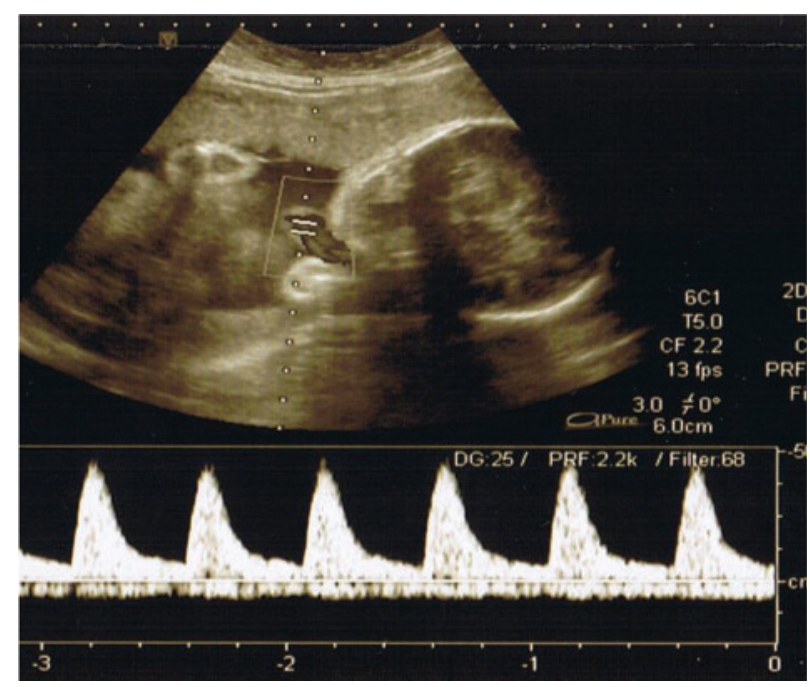

Figure 3. Doppler interrogation of the umbilical artery in the third trimester demonstrating satisfactory end diastolic flow.

twins, an inheritance pattern consistent with new dominant mutations. A suggestion of $X$-linked dominant inheritance was made because no male-to-male transmission was observed in their cases or in a further 21 families documented in previous literature. Apparent autosomal dominant transmission of RSS was subsequently reported in two families. ${ }^{9}$

Cytogenetic reports on RSS indicate the presence of gene defects on chromosomes $1,7,11,17$ and $X^{10-14}$ In patients with intrauterine and postnatal growth restriction, the most commonly reported (10\%) genetic defect is uniparental disomy for chromosome 7 (UPD7) where two copies of maternal chromosome 7 are inherited with no paternal contribution. ${ }^{10}$ Other reported cases show apparently balanced, maternally-inherited reciprocal translocation for chromosome $7,{ }^{11}$ involvement of chromosome $11,{ }^{15}$ chromosome $17,{ }^{12}$ chromosome $1,{ }^{13}$ and $45 \times 0$ karyotype. ${ }^{14}$

RSS does not appear to have greater prevalence in any particular race or ethnic group. It is observed equally in males and females, and is characterised by many varied features with no single finding being pathognomic. The most commonly seen features are severe IUGR, short stature, limb asymmetry, disproportionately large head, frontal bossing, triangular small face, down-turned mouth, micrognathia, clinodactyly and syndactyly of the fingers and/or toes. ${ }^{5,16-19}$ Rarely, the condition may be associated with renal asymmetry and cardiac defects. ${ }^{5,16}$ Generally, cognitive function is considered to be unimpaired, ${ }^{5,18}$ although a cohort of 36 RSS children showed moderate but significant cognitive impairment when IQs were compared with unaffected siblings. ${ }^{20}$

RSS associated intrauterine growth restriction affects both fetal weight and linear growth. 5,6 Therefore, although carried to full term, affected infants are small for gestational age and have low birth weight. Growth restriction and delayed bone development continue post-natally and as a result, affected children exhibit short stature and are typically unusually small and thin for their age. In addition to detecting IUGR, serial ultrasound scans during the third trimester of pregnancy may be helpful in identifying short and/or asymmetric limbs. ${ }^{19}$

Uncommon features of RSS include a high-arched palate, small and crowded teeth, low-set posteriorly rotated and/or prominent ears, unusually high-pitched voice in early years, cryptorchidism, café-au-lait skin spots and short arms. $5,16,19$ These features help distinguish RSS cases from other small for biological age infants.

Diagnosis of RSS is not straightforward and usually rests on a combination of the presence of physical features of RSS and genetic analysis of cells taken at skin biopsy before 5 years of age. Body measurements for asymmetry and radiographic bone age comparisons are also useful; hypoglycemia is compatible with the diagnosis. ${ }^{21}$

Since there are no reliable differentiating sonographic characteristics other than IUGR, prenatal diagnosis of RSS with ultrasound alone is not possible. In addition, because most occurrences are sporadic, it is not possible to identify pregnancies at risk. This further reduces the likelihood of prenatal diagnosis. However, for pregnancies in which early asymmetric intrauterine growth restriction is identified sonographically, prenatal testing for maternal UPD7 is possible with PCR molecular testing of the mother and fetal cells obtained by amniocentesis. Since intrauterine growth restriction often cannot be satisfactorily identified until the third trimester, the options for prenatal diagnosis remain limited. ${ }^{18}$

In our case, in the absence of a definitive test, the multidisciplinary team caring for this patient suspected Down's syndrome as the most likely prenatal diagnosis. Although several studies ${ }^{5,16,19}$ advocate the consideration of RSS in the presence of unexplained IUGR, Down's syndrome has a much higher prevalence. Poor abdominal growth is associated with common genetic abnormalities including trisomies 21, 18 and $13,{ }^{22,23}$ although it is linked more frequently with T18 and T13 than T21. ${ }^{22}$ However, Down's syndrome was still felt to be the most likely diagnosis in view of the suspected cardiac ventricular septal defect which, although refuted pre-natally, was established to be correct when the child was 6 months old. Nyberg et al.'s review of 94 cases of Down's syndrome included five cases with cardiac defects. $^{24}$ In addition, up to 50\% of people with Down's syndrome have cardiac septal defects. ${ }^{25}$ However, it is worth emphasising that cardiac abnormalities are not commonly associated with RSS and this may be coincidental in our case. $^{5}$ There is scant evidence in literature to support including cardiac defects as part of the RSS spectrum although Hansen et al. have described one case with an atrio-septal defect. ${ }^{16}$

Counselling regarding inheritance patterns would be extremely difficult knowing that RSS is genetically heterogeneous and the risk to other family members, including offspring, is not increased over that of the general population. However, counselling that the prognosis for a child with RSS is not bad, particularly when compared with other syndromes which cause IUGR, ${ }^{16}$ and that risk of recurrence is likely to be minimal, is worthwhile. ${ }^{26}$

The management of RSS varies between cases depending on the symptoms of each affected individual.

Owing to poor intake of food, hypoglycaemia should be treated with dietary supplementation, frequent feeding and use of complex carbohydrates. ${ }^{27}$ For the management of severe growth restriction, children may require the administration of artificial growth hormone (recombinant growth hormone). ${ }^{28}$ Those suffering from hypotonia, speech problems and learning disabilities need intervention and individualised educational plans. ${ }^{29}$ Skeletal abnormalities may require surgical orthopaedic intervention. Craniofacial anomalies can be appropriately managed by paediatric dentists in childhood and orthodontists in adolescence. There are a few case reports of rare malignancies, including Wilm's tumour, hepatocellular carcinoma and craniopharyngioma, but children with RSS do not appear to have a significantly increased incidence of neoplasia. 5,30

\section{Conclusion}

RSS is a rare condition representing a spectrum of appearances and symptoms. Most differentiating characteristics are not easily identifiable prenatally at diagnostic ultrasound 
examinations. The symptom most likely to be detected with ultrasound is early asymmetric IUGR. When IUGR is present in the absence of anatomical defects or chromosomal anomalies, we recommend careful serial sonographic evaluation for fetal well being and detailed growth assessments. Although RSS can and should be considered as a possible cause of early asymmetric IUGR, there is little that can be done during the prenatal period. Parents can, however, be reassured that the overall prognosis for a child with RSS is good.

\section{References}

1. Silver H, Kiyasu W, George J, Deamer W. Syndrome of congenital hemihypertrophy, shortness of stature and elevated urinary gonadotrophins. Paediatrics 1953;12:368-375.

2. Russell A. A syndrome of intra-uterine dwarfism recognizable at birth with cranio-facial dysostosis, disproportionately short arms and other anomalies. Proc R Soc Med 1954;47:1040-1044.

3. Jones K. Russell-Silver syndrome. In Smith's recognizable patterns of human malformation. 5th ed. Philadelphia, PA: WB Saunders; 1997, 96-99.

4. Chumei L, Chodirker B, Dawson A, Chudley A. Severe hemihypotrophy in a female infant with mosaic Turner syndrome: a variant of Russell-Silver syndrome? Clin Dysmorphol 2004;13:2, 95-98.

5. Falkert A, Dittmann K, Seelbach-Göbel B. Silver-Russell syndrome as a cause for early intrauterine growth restriction. Prenat Diagn 2005;25:497-501.

6. Gareis FJ, Smith DW, Summitt RL. The Russell-Silver syndrome without asymmetry. J Pediat 1971;79:775-781.

7. Tanner JM, Lejarraga $\mathrm{H}$, Cameron $\mathrm{N}$. The natural history of the Silver-Russell syndrome: a longitudinal study of thirty-nine cases. Pediat Res 1975;9:611-623.

8. Duncan PA, Hall JG, Shapiro LR, Vibert BK. Three-generation dominant transmission of the Silver-Russell syndrome. Am J Med Genet 1990;35:245-250.

9. Al-Fifi S, Teebi A, Shevell M. Autosomal dominant Russell-Silver syndrome. Am J Med Genet 1996;61:96-97.

10. Monk D, Bentley L, Hitchins M, et al. Chromosome 7p disruptions in Silver Russell syndrome: delineating an imprinted candidate gene region. Hum Genet 2002;111:376-387.

11. Dupont JM, Cuisset L, Cartigny M, et al. Familial reciprocal translocation $\mathrm{t}(7 ; 16)$ associated with maternal uniparental disomy 7 in a Silver-Russell patient. Am J Med Genet 2002;111:405-408.

12. Ramirez-Duenas ML, Medina C, Ocampo-Campos R, Rivera H. Severe Silver-Russell syndrome and translocation $(17 ; 20)(q 25 ; q 13)$. Clin Genet 1992;41:51-53.
13. van Haelst $M$, Eussen $H$, Visscher $F$, et al. Silver-Russell phenotype in a patient with pure trisomy 1q32.1-q42.1: further delineation of the pure 1q trisomy syndrome. $\mathrm{J}$ Med Genet 2002;39:582-558.

14. Li CC, Chodirker BN, Dawson AJ, Chudley AE. Severe hemihypotrophy in a female infant with mosaic Turner syndrome: a variant of Russell-Silver syndrome? Clin Dysmorph 2004;13:95-98.

15. Gicquel C, Rossignol S, Cabrol S. Epimutation of the telomeric imprinting center region on chromosome 11p15 in Silver-Russel syndrome. Nat Genet 2005;37:1003-1007.

16. Hansen K, Latson L, Buehler B. Silver-Russell syndrome with unusual findings. Paediatrics 1987;79:1, 125-128

17. Leung A, Fong J, Leong A. Hemihypertrophy. J R Soc Promot Health 2002;122:1, 24-27.

18. Price S, Stanhope R, Garrett C. The spectrum of Silver-Russell syndrome: a clinical and molecular genetic study and new diagnostic criteria. J Med Genet 1999;36:837-842.

19. Wax J, Burroughs R, Wright M. Prenatal sonographic features of Russell-Silver syndrome. J Ultrasound Med 1996;16:253-255.

20. Noeker M, Wollmann H. Cognitive development in Silver-Russell syndrome: a sibling-controlled study. Dev Med Child Neuro 2004;46:340-346.

21. Escobar V, Gleiser S, Weaver D. Phenotypic and genetic analysis of the Silver-Russell syndrome. Clin Genet 1978;13:278-288.

22. Moran C, Tay J, Morrison J. Ultrasound detection and perinatal outcome of fetal trisomies $21,18 \& 13$ in the absence of a routine fetal anomaly scan or biochemical screening. Ultrasound Obstet Gynecol 2002;20:482-485.

23. Vandenbosche RC, Kirchner JT. Intrauterine growth retardation. Am Fam Physician 1998;58:1384-1390, 1393-1394.

24. Nyberg D, Resta R, Luthy D, Hickok DE, Mahony BS, Hirsch JH. Prenatal sonographic findings of Down syndrome: review of 94 cases. Obstet Gynecol 1990;76:370-377.

25. Nyberg D, McGahan J, Pretorius D, Pilu G. Chromosomal abnormalities. In Diagnostic imaging of fetal anomalies. Philadelphia, PA: Lippincott Williams \& Wilkins, 2003, 874.

26. Wollmann HA, Kirchner T, Enders H, Preece MA, Ranke MB. Growth and symptoms in Silver-Russell syndrome: review on the basis of 386 patients. Eur J Pediatr 1995;154:958-968.

27. Azcona C, Stanhope R. Hypoglycaemia and Russell-Silver syndrome. J Pediatr Endocrinol Metab 2005;18:663-670.

28. Christoforidis A, Maniadaki I, Stanhope R. Managing children with Russell-Silver syndrome: more than just growth hormone treatment? J Pediatr Endocrinol Metab 2005;18:651-652.

29. Lai KY, Skuse D, Stanhope R, Hindmarsh P. Cognitive abilities associated with the Silver-Russell syndrome. Arch Dis Child 1994;71:490-496.

30. Draznin MB, Stelling MW, Johanson AJ. Silver-Russell syndrome and craniopharyngioma. J Pediatr 1980;96:887-889. 